\title{
Lutetium Lu 177 Lilotomab-satetraxetan
}

National Cancer Institute

\section{Source}

National Cancer Institute. Lutetium Lu 177 Lilotomab-satetraxetan. NCI Thesaurus. Code C162565.

A radioconjugate consisting of lilotomab, a murine immunog lobulin G1 (IgG1) antibody directed against the CD37 antigen, conjug ated via the chelating agent 2-(4isothiocyanatobenzyl)-1,4,7,10-tetraazacyclododecane-tetraacetic acid (p-SCN-BnDOTA) with potential antineoplastic activities. Upon administration of lutetium Lu 177 lilotomab-satetraxetan, the lilotomab moiety binds to CD37 expressed on certain tumor cells. Upon binding, lutetium Lu 177 lilotomab-satetraxetan delivers a cytotoxic dose of beta radiation to CD37-expressing cells. CD37 is a transmembrane glycoprotein expressed at high-levels on B-cells and to a lesser extent on T-cells and myeloid cells, and is frequently overexpressed in certain B-cell lymphomas. 